\title{
HEMORRHAGIC SHOCK: DEFINITION AND CRITERIA FOR ITS DIAGNOSIS
}

\author{
BY HAROLD C. WIGGERS AND RAYMOND C. INGRAHAM \\ (From the Department of Physiology, University of Illinois, College of Medicine, Chicago)
}

(Received for publication May 21, 1945)

The term hemorrhagic shock as employed in the medical literature connotes a variety of unqualified conditions, reversible and irreversible, which follow the loss of variable and frequently unknown quantities of blood. Further confusion has resulted from reports in which supposedly panacean measures for the treatment of these variable conditions are described. Unfortunately, since the existing condition of the animal or patient at the onset of treatment is seldom clearly defined, it has become increasingly difficult to evaluate the efficacy of the various remedies for practical purposes. It is of paramount importance that authors more rigidly define the conditions which are referred to under the heading of hemorrhagic shock. Thus, as a start in this direction, we shall initially define our connotation of the condition which will be discussed from a diagnostic viewpoint later in this paper.

Experience gained from an extended series of hemorrhagic-hypotension studies ( 1 to 3 ) permits the formulation of a simple classification for the conditions experienced by dogs and presumably by man following different degrees of rapid hemorrhage. (1) Losses of blood up to 30 to 40 per cent of the total blood volume, for instance, induces a hypotensive state from which a normal, healthy animal will usually recover in time with no treatment other than that inherently afforded by automatically regulated compensatory mechanisms $(4,5)$. This condition may be designated as a simple hemorrhagic-hypotensive state. (2) Where the loss of blood exceeds 40 per cent of the circulating blood volume and if acute cardio-respiratory failure does not supervene, a sustained hypotension develops which leads to progressive tissue anoxia and irreversible damage. Recovery from this state is possible if this progressive deterioration is arrested by the timely infusion of suitable agents in adequate amounts. This condition may be designated as the impending shock state. (3) Failure to check the latter condition permits transition to an irreversible shock state, the existence of which can be diagnosed at its onset by the observance of several physiological manifestations to be discussed.

Having thus indicated our interpretation of the hemorrhagic shock state as an irreversible condition, we are now in position to consider those criteria which permit diagnosis of its existence.

\section{PROCEDURE}

Experiments on 39 dogs, subjected to a routine standardized hemorrhagic-hypotension procedure described elsewhere in detail $(1,2)$ yielded the data to follow. Briefly, rapid hemorrhaging via a femoral artery was continued until the mean arterial blood pressure descended to $40 \mathrm{~mm}$. $\mathrm{Hg}$. This hypotensive level was sustained for 90 minutes by further withdrawals of blood when necessary. Thereupon, all withdrawn blood was reinfused via a femoral vein. Local novocaine anesthesia was employed at operative sites for arterial and venous cannulation. The anticoagulant, Liquaemin, 1 was used in all bleeding procedures.

\section{RESULTS AND DISCUSSION}

The first 2 physiological manifestations to be discussed permit diagnosis of the irreversible shock state during the hypotension period; the remaining 3 serve this purpose early in the postreinfusion period. The significance of the shock criteria becomes apparent in comparing the contemporaneous characteristics observed in dogs which survived and in those which did not. Twelve survived and 27 died. Among the latter, 5 animals lived from 24 to 72 hours, following reinfusion of withdrawn blood. In the other 22 dogs, the post-reinfusion survival times were considerably shorter. It was not possible to detect significant diagnostic shock criteria, functional or post mortem, in the 5 dogs with prolonged postreinfusion survival times, but for want of positive evidence of other possible causes of death, they

1 We are indebted to Roche Organon, Inc., for their generous supply of Liquaemin used in this study. 
are included in the irreversible-shock group. Most of the following criteria when encountered severally are usually adequate for diagnosis of a prevailing irreversible-shock state. It is advisable, however, to seek two or more such criteria to establish the diagnosis with certainty.

\section{(1) Spontaneous arterial pressure decline}

If, during the hypotension period, the arterial blood pressure distinctly begins to decline below the established level ( 40 to $45 \mathrm{~mm}$. $\mathrm{Hg}$ ) and continues to do so despite restoration to this level with the aid of an infusion of a small volume of blood or saline, it is reasonably certain that the irreversible shock state prevails. Immediate reinfusion of all withdrawn blood at this phase of the experiment usually reproduces a very satisfactory arterial blood pressure which, along with the reasonably normal outward appearance and behavior tends to mask the truly critical shock state which prevails. Such prompt restoration of the original blood volume has never provided other than this apparent, temporary circulatory benefit to the 22 animals in which the spontaneous arterial pressure decline was detected during hypotension. On the other hand, this decline never occurred in the 12 animals which survived the hemorrhagic procedures; in fact, there was almost always a persistent and opposite tendency for blood pressure to ascend above the established hypotension level. In the remaining 5 fatalities with the extraordinarily prolonged post-reinfusion survival times, the declining tendency was not distinctly recognizable. Under pressure, it was possible, in some instances, to imagine that the tendency might be present in the terminal moments of the hypotension period. These animals appear to be borderline cases between the two extremes encountered in the other dogs. Yet, they finally succumbed in what has been designated as irreversible shock.

The recognizable occurrence, therefore, of this phenomenon appears to signify that the transition from the adequately compensated impending shock state to the inadequately compensated irreversible shock state has occurred. The moment or process of transition is undetectable but appears to occur within a 10-minute interval. Consequently, when recognized, it permits diagnosis of an existing shock state with an extremely unfavorable prog- nosis in the present state of our knowledge of treatment.

\section{(2) Relative hemoconcentration during the hypotension period}

In 26 of these dogs, a study of blood and plasma specific gravity changes was conducted on blood samples drawn at arbitrarily selected intervals throughout the course of the control, hypotension, and post-reinfusion periods. The Barbour and Hamilton method for the determination of specific gravity was employed (6). In most instances the blood and plasma specific gravity changes paralleled one another, any slight differences being related to occasional augmentation of splenic activity in these unanesthetized dogs.

Since there is good evidence that no significant loss of plasma proteins from the circulation occurs during the period of low arterial blood pressure $(1,7)$, it seems reasonable to conclude that any reduction in specific gravity reflects primarily the marked degree of auto-infusion which occurs in well-hydrated animals. Because of the parallel behavior of blood and plasma specific gravity changes, it seems permissible in succeeding discussion to use the terms plasma dilution and hemodilution, as well as plasma concentration and hemoconcentration interchangeably.

Eight dogs never transcended from the impending shock state to the irreversible shock state and hence, they survived indefinitely. In these animals, plasma dilution of varying degree was commonly observed during the initial rapid bleeding period. In some (curves $\mathrm{C}$ and $\mathrm{D}$ of Figure 1), this process continued throughout the entire or major portion of the 90-minute hypotension period. In the few in which complete arrest of the plasma dilution was noted, the status quo of maximal dilution was maintained until the period of hypotension was terminated. In no instance was a shift toward relative hemoconcentration even remotely suggested.

In 15 dogs which underwent the transition from impending to irreversible shock and which usually died within 10 hours following the return of all withdrawn blood, the plasma dilution process was always arrested after 60 minutes of hypotension (see curves A and B of Figure 1 as typical examples) and occasionally by the time $30 \mathrm{~min}$ utes had elapsed. Within the first 60 minutes, 4 


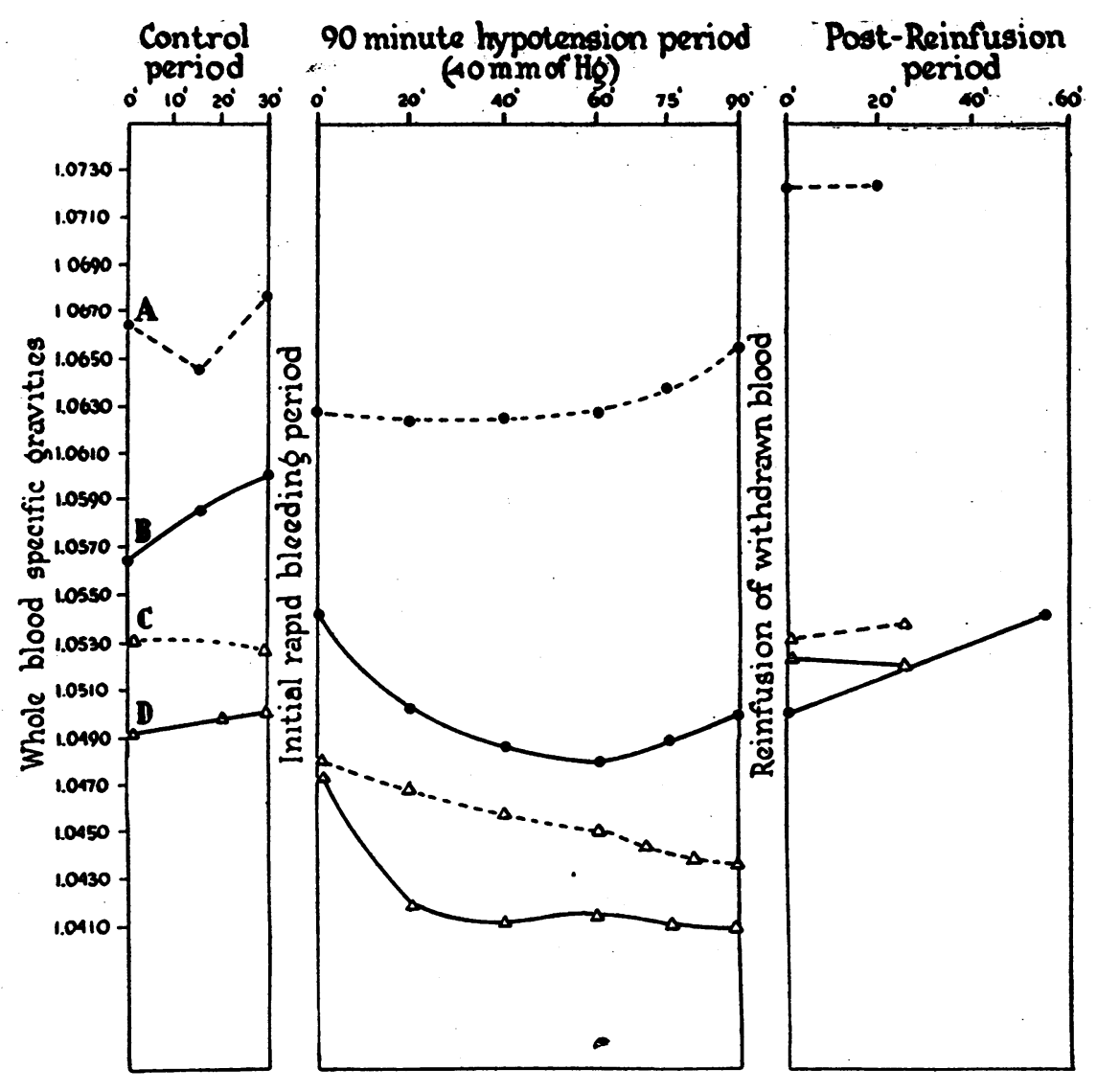

FIG. 1

Curves A and B are selected as typical examples of the blood specific gravity changes seen in 2 dogs who died in shock after reinfusion of withdrawn blood, Curves $C$ and $D$ depict similar changes in 2 dogs who survived the hemorrhage following reinfusion of withdrawn blood.

of these dogs began in addition to reveal definite tendencies toward relative hemoconcentration, and along with 5 others, showed a marked degree of relative hemoconcentration as the hypotension period was terminated. Curves A and B of Figure 1 depict neither the least nor most severe relative hemoconcentration seen in these animals but are considered as typical examples. In the other 6 dogs of this group, the maximal dilution attained early in the hypotension period remained at status quo until the withdrawn blood was reinfused.

Persistent plasma dilution tendencies were also encountered in 3 dogs whose post-reinfusion survival times were exceptionally long (72, 60 and 48 hours). No tendencies toward relative hemoconcentration were observed. Again, these long survival animals appear to represent borderline cases which defy easy diagnosis.
Under the conditions of these experiments, it is usually safe to assume that any animal in which hemodilution has persisted for 80 to 90 minutes is still in the impending shock state and is likely to survive indefinitely following the restoration of the original blood volume. In such animals, the other positive criteria for the diagnosis of the irreversible shock state are never observed. On the other hand, one can, with a greater degree of positiveness, diagnose a prevailing irreversible shock state with its accompanying fatal prognosis, when plasma dilution is arrested early and a relative degree of plasma concentration ensues. Furthermore, the outlook may be considered definitely unfavorable when dilution of the blood is arrested within the first 60 minutes of the hypotension period, even though no definite trend toward plasma concentration can be detected. In such 
instances, however, a diagnosis of prevailing irreversible shock should be substantiated by other obvious criteria.

\section{(3) Diarrhea and passage of bloody-tinged fecal material}

It was not uncommon to witness successful and unsuccessful attempts to execute the act of defecation during the experimental period, even though the animals were in an unnatural supine position. Presumably, the prepotent stimulus for the act arose from the reduction of intestinal blood flow and the accompanying asphyxial conditions in that region. Such evacuation was much more violent and distinctive upon their release from the restricted position following completion of reinfusion and surgical closure of operative sites. In the animals which survived indefinitely, the feces were usually well formed and of essentially normal consistency. In addition, one good evacuation usually sufficed. Among the fatalities, including those with long post-reinfusion survival times, it was quite common to observe a persistent and extremely fluid, "bloody" diarrhea. It was nearly always possible to distinguish those animals which would survive the procedure from those which would succumb in shock on the basis of this observation.

\section{(4) Post-reinfusion heart rate}

Another criterion by means of which a prevailing irreversible shock state can be diagnosed, irrespective of the arterial blood pressure, entails a comparison of the relationship between the control and the post-reinfusion (P-R) heart rate. It is expedient to delay the consideration of this criterion momentarily in order to mention briefly another related physiological phenomenon encountered in nearly every animal.

During and following the reinfusion of all withdrawn blood and its accompanying elevation of arterial blood pressure from the hypotensive level, even the more active and restless animals suddenly became quiet and apparently sleepy. They seldom aroused from this apparent soporific or sedative state until removed from the table and allowed to roam about the laboratory, at which time complete wakefulness and initiative seemed restored. This sudden somnolence might be attributed to a rise in the intra-cranial pressure associated with the rapid elevation of systemic blood pressure. This is not a wholly adequate explanation, inasmuch as the soporific effect occurred in some dogs before much blood had been administered and prior to any significant elevation of blood pressure. Regardless of the ultimate explanation of this interesting phenomenon, it stands to reason that in this quiescent, unemotional state, heart rates nearer to basal values should be anticipated than those existing initially under control conditions. Consequently, excluding disturbing influences of internal origin, conditions favor equal or slower P-R heart rates than control rates, since the latter are frequently subject to prevailing acceleratory emotional factors. P-R heart rates were counted within 30 minutes of the completion of reinfusion.

Returning now to consider the actual P-R heart rates, it is seen that they were essentially equivalent to the respective control rates in the dogs which survived indefinitely (Figure 2A). Among the few whose P-R rates exceeded the controls, the most rapid was a rate of 135 beats per minute. In those which did not survive, however, the P-R heart rate usually exceeded the control (Figure 2B). This appears to be contradicted in the first 3 experiments of this group and in 1 other, in which the P-R and control rates were quite similar. From the graph of the post-reinfusion survival times (Figure 2B), however, it is noticed that the first 3 animals belong to the "borderline" shock group with the exceptionally long postreinfusion survival times. There is little reason to doubt that marked acceleration would have been encountered in these dogs, had the heart rate values been obtained during the more terminal stage of their survival period.

In these hemorrhagic-hypotension studies, in which considerable care was taken to eliminate environmental acceleratory influences, a P-R heart rate of 150 or more beats per minute correlated perfectly as a reliable diagnostic indication of prevailing irreversible shock, despite the fact that the mean blood pressure usually exceeded $110 \mathrm{~mm}$. $\mathrm{Hg}$ and several were as high as $150 \mathrm{~mm}$. $\mathrm{Hg}$. A correct diagnosis and prognosis of fatality based upon this criterion alone in the absence of other positive criteria proved infallible in several in- 


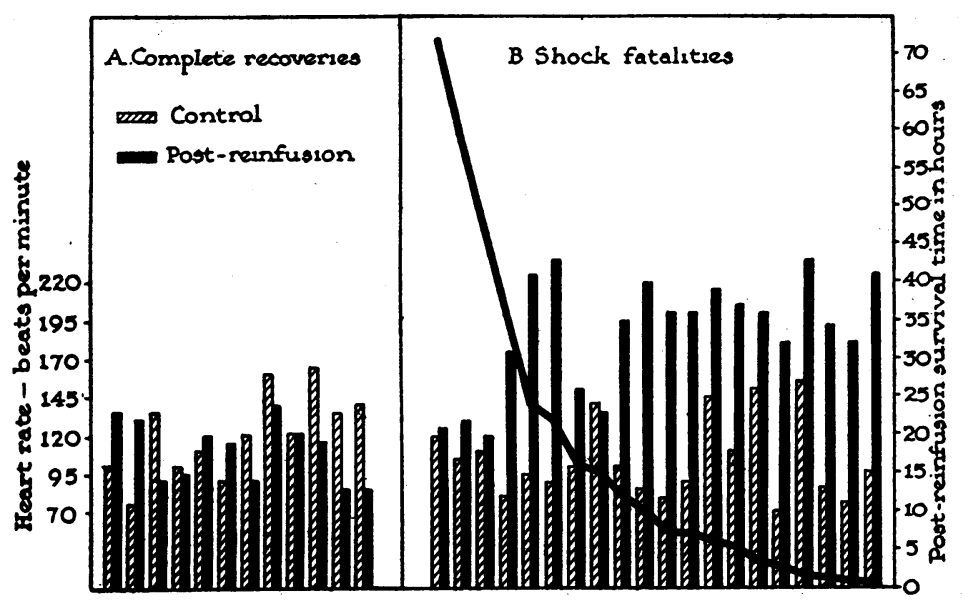

Fig. 2. Individual Hemorrhagic-Hypotension Shock Experiments

The curve in (B) above depicts the relationship between the length of the post-reinfusion survival times of the various animals to their respective control versus post-reinfusion heart rates. Those with significant differences in the latter comparison lived the least amount of time after reinfusion of all withdrawn blood.

stances. A similar shock diagnosis will usually prove correct whenever P-R heart rates are significantly faster than the respective control rate.

\section{(5) Post-reinfusion blood or plasma specific gravity}

It is also possible to diagnose whether shock is present by comparing the plasma or blood specific gravity value determined approximately $30 \mathrm{~min}$ utes after the reinfusion of withdrawn blood with the previously obtained control value. In 8 dogs which survived indefinitely, the P-R plasma specific gravity was essentially equivalent to, or lower than, the corresponding control value (Figure $3 \mathrm{~A})$. Among the 18 fatalities in which these determinations were made, 12 exhibited P-R plasma values significantly exceeding respective control figures; 2 showed equivalent $P-R$ and control values; whereas, in the remaining 4 dogs, they were still lower than the controls, as in the case of many recovery animals (Figure 3B). In the latter 6 dogs in which P-R plasma specific gravities did not exceed control values, the postreinfusion survival times were usually longer than in the other fatalities $(72,60,48,24,12$, and 5 hours). It seems reasonable to interpret the usual increase in plasma and blood specific gravity seen after the reinfusion as an expression of plasma or hemoconcentration. This phenomenon is not related to the specific gravity of the reinfused blood, inasmuch as the latter was always lower than the control value, as might be expected on the basis of the plasma dilution which occurs during bleeding. Just how this hemoconcentration occurs in the face of an obvious loss of plasma and blood cells through intestinal capillaries (free blood in the intestinal lumen) is not clearly understood. Regardless of the explanation, the elevation of $\mathrm{P}-\mathrm{R}$ plasma specific gravity above control values may be regarded as a reliable criterion upon which to base a diagnosis of prevailing irreversible shock.

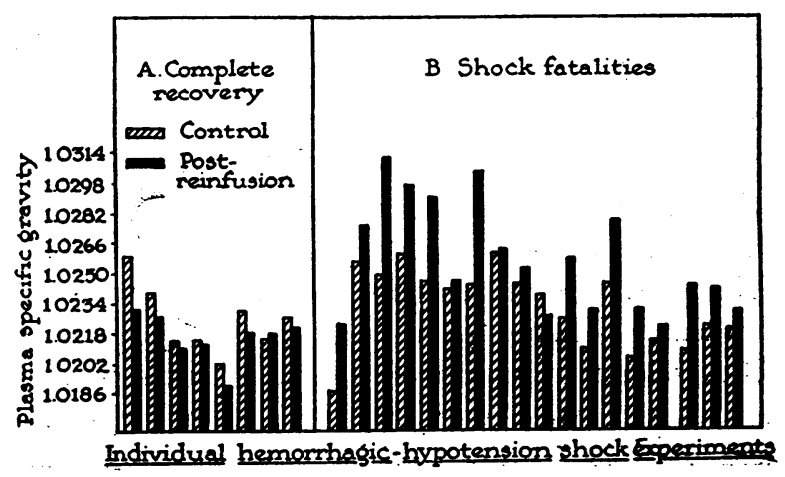

Fig. 3. Individual Hemorrhagic-Hypotension SHOCK EXPERTMENTS 


\section{GENERAL DISCUSSION}

Conditions following hemorrhages of variable duration and intensity range from rather insignificant physiological disturbances to the extremely critical situations generally referred to as shock. There appears, however, to be little agreement as to the terminologies for the various recognizable stages which occur within this range. The greatest discrepancies in terminology are evident in the reports of the laboratory experimentalist and the internist. This is understandable and not wholly attributable to the fact that the observations are made upon different species. Unaided by "control" observations, the clinician is faced with the task of estimating the progress of events from the incidence of hemorrhage until he sees the patient. He may have little knowledge of the volume of blood lost or of the length of time that hemorrhagic-hypotension has been exerting its deleterious effects. From the experimental viewpoint, he is further handicapped in that he is not justified in allowing the condition to progress further than status quo. He must render immediate treatment, thus denying him further information of the progressive events which might follow. It is, a priori, easy to understand the difficulty experienced in comparing such disturbances in patients with those which permit more elaborate and unhampered observation in animals, and in the attempts to arrive at terminologies for the expression of essentially equivalent conditions. An apparent impasse of this nature, need not, however, interfere with further progress towards a standardized classification of the principal hemorrhagic stages in man and animal. In the introduction, we have offered a simplified classification as an initial step in this direction with the purpose in mind of eliminating the application of the term "shock" in all simple hemorrhagic conditions and of calling attention to the necessity of delineating the stage of progressive deterioration to the ensuing irreversible shock state.

It must, for instance, be recognized that animals undergoing a 30 to 40 per cent reduction of their total blood volume, will, if healthy and restrained in a resting position, slowly recover without any treatment at the hands of the investigator. The term shock should never be applied to this simple uncomplicated hemorrhagic condition. For want of a better term, we have designated this condition a simple hemorrhagic hypotension state. Suitable infusions at any time will lead to prompt restoration of essentially normal conditions.

If, as a result of greater loss of blood or other complicating factors, a more severe and prolonged period of hypotension results, the stage is set for increasing tissue anoxia and hence, permanent tissue damage. If the blood volume is restored in these animals after one hour of severe hypotension $(40 \mathrm{~mm} . \mathrm{Hg})$, they usually recover (1). Extension of the hypotension period for an additional 30 minutes leads, in most animals, to the appearance of criteria (persistent decline of blood pressure, relative hemoconcentration) which indicate that survival is unlikely upon restoration of the original blood volume; i.e., an irreversible condition has been attained. This condition was designated as irreversible hemorrhagic shock to distinguish it from the preceding condition from which it developed (impending shock state) in which none of the diagnostic criteria were observed. Thus, the restoration of the original blood volume at any time during impending shock leads to recovery if extraneous complications are not involved. If a careful examination of the literature is made, it will be recognized that the various panacean measures found effective in the treatment of "so-called hemorrhagic shock" were actually administered under circumstances which correspond either to the simple hemorrhagic-hypotension state or the impending shock state. None of those called to our attention mention the occurrence of the criteria which permit diagnosis of the true shock state.

The authors have frequently asked and sought an experimentally documented answer to this question. Does continuation of the untreated impending shock state lead directly to the irreversible shock state, with its accompanying detectable manifestations, or is there a distinguishable brief, intermediate "reversible shock state"? We have been unable to obtain any evidence of transition through an intermediate stage of this nature.

It is also expedient to re-emphasize that these transitional stages are not clearly demarcated in all animals. As in other disorders, there are borderline cases in which the irreversible shock state apparently prevails without recognition until just before death (dogs with long post-reinfusion 
survival times). On the other hand, one must not be misled into a favorable prognosis on the basis of satisfactory blood pressures or apparently normal behaviour characteristics. Many of our animals which succumbed resembled those which recovered so far as these aspects are concerned. A good post-reinfusion blood pressure often masked the existing irreversible shock state.

In conclusion, it seems reasonable to assume that the terminologies suggested above should suffice to delineate the three major, distinguishable conditions which are likely to develop after sizeable hemorrhages which vary considerably in duration and intensity.

\section{SUM MARY}

An attempt is made to differentiate 3 major conditions which may develop from uncomplicated hemorrhages of variable duration and intensity. These and their suggested terminologies are as follows: (a) a simple hemorrhagic hypotension state, from which recovery is almost assured without treatment in the form of transfusions or infusions. (This is by definition; withholding of treatment is not suggested.) (b) More severe hemorrhages lead to an impending shock state with severe, sustained hypotension and consequent tissue anoxia. If uncorrected within a certain interval, (c) a third condition develops which is designated as the irreversible shock state. The existence of this state may not be recognized at its onset unless one looks for specific accompanying diagnostic criteria. Five such criteria, definitely diagnostic for the conditions under which shock was produced in this study, are discussed in detail. The transition from the impending to the irreversible shock state occurred during the 90 -minute hypotension period $(40 \mathrm{~mm}$. $\mathrm{Hg}$ ) usually during the last 15 minutes.
Two criteria (persistent decline of blood pressure below $40 \mathrm{~mm}$. $\mathrm{Hg}$, relative hemoconcentration) permitted diagnosis of existing irreversible shock during the hypotension period. The other 3 criteria enabled this state to be recognized, if present, early in the post-reinfusion period. These three were: (a) rapid heart rate in relation to the control heart rate; (b) passage of bloody and fluid fecal material; and (c) actual hemoconcentration. Most animals in irreversible shock revealed three or more of these diagnostic manifestations. There were a few borderline cases, however, in which irreversible shock prevailed but could not be diagnosed because none of the criteria were detected. These animals lived for long periods following the termination of the hypotension period and the reinfusion of all withdrawn blood.

\section{BIBLIOGRAPHY}

1. Wiggers, H. C., Ingraham, R. C., and Dille, J., Hemorrhagic-hypotension shock in locally anesthetized dogs. Am. J. Physiol., 1945, 143, 126.

2. Ingraham, R. C., and Wiggers, $H$. C., The value of supplemental alkalinizing therapy in hemorrhagic shock. Fed. Proc., 1945, 4, 36.

3. Ingraham, R. C., and Wiggers, H. C., Alkalinizing agents and fluid priming in hemorrhagic shock. Am. J. Physiol., 1945, 144, 505.

4. Walcott, W. W., Standardization of and blood volume in experimental hemorrhagic shock. Am. J. Physiol., 1945, 143, 247 and 254.

5. Ivy, A. C., Greengard, H., Stein, I. F., Grodins, F. S., and Dutton, P. F., The effect of various blood substitutes in resuscitation after otherwise fatal hemorrhage. Surg., Gynec. and Obst., 1943, 76, 85.

6. Barbour, H. C., and Hamilton, W. F., The falling drop method for determining specific gravity. J.A.M.A., 1927, 88, 91.

7. Fine, J., and Seligman, A. M., Traumatic shock. IV. A study of the problem of "lost plasma" in hemorrhagic shock by the use of radioactive plasma protein. J. Clin. Invest., 1943, 22, 285. 